\title{
A Randomized Double-blind Placebo-controlled Trial on the Effect of Magnesium Oxide in Patients With Chronic Constipation
}

\author{
Sumire Mori, ${ }^{1}$ Toshihiko Tomita, ${ }^{1 *}$ Kazuki Fujimura, ${ }^{1}$ Haruki Asano, ${ }^{1}$ Tomohiro Ogawa, ${ }^{1}$ Takahisa Yamasaki, ${ }^{1}$ Takashi Kondo, \\ Tomoaki Kono, ${ }^{1}$ Katsuyuki Tozawa, ${ }^{1}$ Tadayuki Oshima, ${ }^{1}$ Hirokazu Fukui, ${ }^{1}$ Takeshi Kimura, ${ }^{2}$ Jiro Watari, ${ }^{1}$ and Hiroto Miwa ${ }^{1}$ \\ ${ }^{I}$ Division of Gastroenterology, Department of Internal Medicine, Hyogo College of Medicine, Nishinomiya, Hyogo, Japan; and ${ }^{2}$ Department of \\ Pharmacy, Hyogo College of Medicine, Nishinomiya, Hyogo, Japan
}

\section{Background/Aims}

Magnesium oxide (MgO) has been frequently used as a treatment for chronic constipation (CC) since the 1980s in Japan. The aim of this study is to evaluate its therapeutic effects of $\mathrm{MgO}$ in Japanese CC patients.

\section{Methods}

We conducted a randomized, double-blind placebo-controlled study. Thirty-four female patients with mild to moderate constipation were randomly assigned to either placebo $(n=17)$ or $\mathrm{MgO}$ group $(n=17) 0.5 \mathrm{~g} \times 3 /$ day for 28 days. Primary endpoint was overall improvement over the 4-week study period. Secondary endpoints were changes from baseline in spontaneous bowel movement (SBM), response rates of complete spontaneous bowel movement (CSBM), stool form, colonic transit time (CTT), abdominal symptom, and quality of life.

\section{Results}

One patient failed to complete the medication regimen and was omitted from analysis: data from 16 placebo and $17 \mathrm{MgO}$ patients were analyzed. The primary endpoint was met by $25.0 \%$ of placebo vs $70.6 \%$ of $\mathrm{MgO}$ group ( $P=0.015)$. MgO significantly improved SBM changes compared to placebo $(P=0.002)$. However, $\mathrm{MgO}$ did not significantly improved response rates of CSBM compared to placebo $(P=0.76)$. In addition, $\mathrm{MgO}$ significantly improved Bristol stool form scale changes $(P<0.001)$ and significantly improved CTT compared to the placebo group $(P<0.001)$. MgO significantly improved the Japanese version of the patient assessment of constipation quality of life $(P=0.003)$.

\section{Conclusion}

Our placebo-controlled study demonstrated that $\mathrm{MgO}$ was effective treatment for improving defecation status and shortened CTT in Japanese CC patients with mild to moderate symptoms.

(J Neurogastroenterol Motil 2019;25:563-575)

\section{Key Words}

Constipation; Defecation; Double-blind method; Japan; Magnesium oxide

Received: November 13, 2018 Revised: March 15, 2019 Accepted: July 20, 2019

(a) This is an Open Access article distributed under the terms of the Creative Commons Attribution Non-Commercial License (http://creativecommons. org/licenses/by-nc/4.0) which permits unrestricted non-commercial use, distribution, and reproduction in any medium, provided the original work is properly cited.

*Correspondence: Toshihiko Tomita, MD, PhD

Division of Gastroenterology, Department of Internal Medicine, Hyogo College of Medicine, 1-1, Mukogawa-cho, Nishinomiya, Hyogo 663-8501, Japan Tel: +81-798-45-6665, Fax: +81-798-45-6661, E-mail: tomita@hyo-med.ac.jp 


\section{Introduction}

Chronic constipation (CC) is neither a symptom nor a disease; rather, it is a state of congested feces in the large intestine due to an infrequent and low quantity of bowel movements or a state of being unable to pass the feces from the large intestine in a comfortable manner. ${ }^{1-8}$ Delayed colonic transit time (CTT) is considered as one of the main etiological mechanisms of constipation. ${ }^{9-10}$

Constipation is a common condition encountered in all clinical departments, and is known to markedly diminish activities of daily living and labor productivity and lessen the quality of life (QOL). Thus, it is a condition that should be treated quickly and appropriately.

The main drugs used in Japan to treat constipation include osmotic laxatives (saline or sugar laxatives), drugs that alter epithelial function, stimulant laxatives, bulk-forming laxatives, and herbal medicines. Among these, the osmotic laxative magnesium oxide (MgO) has been frequently used in Japan since the 1980s. It is currently used by approximately 10 million patients in Japan. ${ }^{11}$ It is known to be an excellent laxative that can be taken by anyone owing to its low cost, ease of dose adjustment, and ease of use.

When $\mathrm{MgO}$ enters the stomach, it first reacts with gastric acid and becomes magnesium bicarbonate or magnesium carbonate in the small intestine. It absorbs water from the intestinal walls and the intestinal content expands from the water. This intestinal stimulation is then thought to induce bowel movement. As $\mathrm{MgO}$ can lead to hypermagnesemia in elderly people and patients with impaired renal function, the serum magnesium level needs to be measured regularly. However, $\mathrm{MgO}$ is inexpensive, the dose can be easily adjusted, and it is non-habit forming. Thus, it is currently the most frequently used medicine and is considered a superior, easy-to-use laxative for any patient.

In the constipation clinical practice guidelines in Japan, prescription of osmotic laxatives and drugs that alter epithelial function is the most strongly recommended for the treatment of $\mathrm{CC}$. $^{12}$ These drugs are highly regarded with evidence level $A$, and are the first-line treatment for constipation. However, aside from a report published in the 1990s that examined the effect of $\mathrm{MgO}$ in an open-label physician-led questionnaire investigation, to date, no randomized clinical trials have examined the use of $\mathrm{MgO}{ }^{13}$

Therefore, in the present study, we evaluate the therapeutic effects of $\mathrm{MgO}$ in an objective and scientific manner, using a patient self-administered questionnaire. The findings obtained from this study are also important from a clinical perspective, since patient- reported outcome (PRO) evaluations were performed with appropriate measures to exclude bias whenever possible. ${ }^{14,15}$

\section{Materials and Methods}

\section{Study Design}

This placebo-controlled double-blind study was conducted at a single center. We recruited 34 patients with functional constipation (FC) who visited the outpatient department of our hospital between September 2017 and April 2018. ${ }^{16}$ After written informed consent was obtained from all patients, they were randomly assigned to receive either $\mathrm{MgO}$ ( $\mathrm{MgO}$ group; 17 patients, $0.5 \mathrm{~g} \mathrm{MgO} /$ capsule orally) or placebo (placebo group; 17 patients, 0.5 g placebo capsule orally) 3 times per day after meals for 28 days (4 weeks) (Fig. 1). The randomization code was concealed until the end of the trial. The $\mathrm{MgO}$ and placebo capsules were made by filling the capsules with $\mathrm{MgO}$ and lactose, respectively. We performed all experiments in accordance with human ethics regulations (Hyogo College of Medicine: approval No. 2711). This clinical study is registered with the University Hospital Medical Information Network Clinical Trials Registry (No. UMIN000028973). The trial was conducted according to the principles governing human research in the Declaration of Helsinki. All authors had access to the study data and reviewed and approved the final manuscript.

\section{Patients}

The study enrolled 34 female patients. Average patient age was $40.6 \pm 12.8$ years at the start of the study. This study selected patients with mild to moderate FC that met the Rome IV diagnostic criteria. ${ }^{16}$ Patients with mild to moderate constipation were defined as those who are eligible but do not need medication on a daily basis, or who took over-the-counter (OTC) laxatives as needed. Patients also had to be able to understand the contents of the study

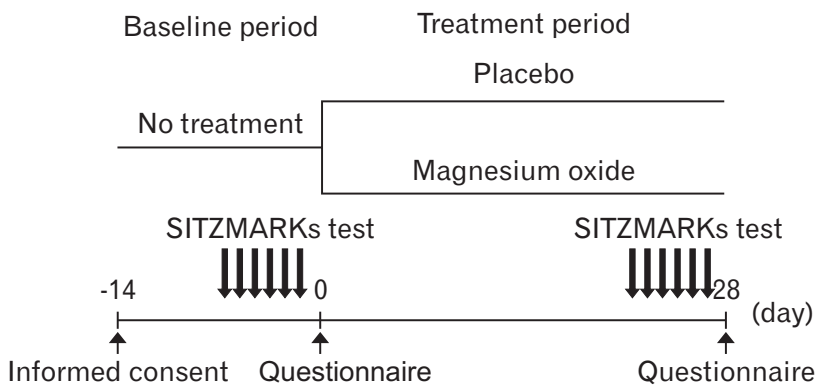

Figure 1. Study design. 
and provide consent to participate.

The exclusion criteria of this study were as follows; (1) age < 20 years or $>75$ years of age, (2) being prescribed laxatives from medical institutions like hospitals or clinics, (3) using OTC laxatives 6-7 times/week, (4) finding it difficult to stop using the OTC drugs that they were already taking after providing consent to participate in this study, (5) patients with kidney disorder, (6) patients with impaired cardiac function, hypermagnesemia, ${ }^{11,17}$ (7) patients who have taken gastric secretion inhibitors, ${ }^{18}(8)$ patients who may become pregnant, (9) patients who thought to have secondary constipation, (10) patients who have taken drugs that might interact with $\mathrm{MgO}$, and (11) patients who are considered unsuitable for participation by the physician in charge.

\section{Outcome Measures}

The primary endpoint was overall improvement of $\mathrm{MgO}$ compared with those of the placebo by evaluating symptoms before and after study drug administration in each group. Secondary endpoints were changes in average spontaneous bowel movement (SBM) during the treatment period, response rate of complete spontaneous bowel movement (CSBM), change in average Bristol stool form scale (BSFS) score during the treatment period, CTT before and after taking the medication, change in abdominal symptoms (abdominal bloating, abdominal discomfort, straining during defecation, and sensation of incomplete evacuation), health-related QOL short form-8 (SF-8) questionnaire scores ${ }^{19,20}$ and defecation-related QOL scores assessed using the Japanese version of the patient assessment of constipation QOL (JPAC-QOL) questionnaire, ${ }^{21-23}$ and changes in the scores evaluated by a constipation scoring system (CCS) ${ }^{24}$ a general evaluation of constipation, between the $\mathrm{MgO}$ and placebo groups.

\section{Colonic Transit Time}

CTT was measured by the continuous marker method using colon-opacifying markers (Sitzmarks, Konsyl Pharmaceuticals, Fort Worth, TX, USA). The patients took 1 capsule per day containing 20 markers, and CTT was measured on the 7th day using X-rays to detect the number of markers in the colon. ${ }^{25}$ We also used the segmental method to evaluate the transit time for the right colon, the left colon, and the rectosigmoid colon separately. ${ }^{26}$

\section{Questionnaires (Defecation Diary, Symptoms, Quality of Life, and Constipation Scoring System)}

We developed an original defecation diary and questionnaire to evaluate the response rate of overall improvement in constipation symptoms, weekly mean SBMs over 4 weeks, change in weekly mean and mean values during the treatment period, weekly mean CSBM and the changes in its weekly mean value and response rate, and constipation symptoms. Overall improvement was evaluated using a 5 point scale (1: significantly improved; 2: improved; 3: slightly improved; 4: unchanged; and 5: exacerbated), and the response rate of overall improvement was defined as the percentage of patients who had scores of 1 or 2 for 2 or more of the 4 evaluation weeks. ${ }^{13}$ The mean value during the treatment period was the mean of weekly mean values over the 4 weeks. CSBM response was defined as a weekly mean of 3 or more instances of SBM with no sensation of incomplete evacuation, or an increase by 1 or more instance of SBM with no sensation of incomplete evacuation compared to baseline for 2 or more of the 4 evaluation weeks. ${ }^{27,28}$ Stool form was evaluated on a 7-stage scale using the BSFS (1: separate hard lumps, like nuts [hard to pass]; 2: sausage-shaped but lumpy; 3: like a sausage but with cracks on the surface; 4: like a sausage or snake, smooth and soft; 5: soft blobs with clear cut edges [passed easily]; 6: fluffy pieces with ragged edges, a mushy stool; and 7: watery, no solid pieces [entirely liquid]), ${ }^{29,30}$ and the changes in their weekly mean and mean values during the treatment period were evaluated. With regard to constipation symptoms, the intensity of abdominal distension, abdominal discomfort, sensation of incomplete evacuation, and straining during defecation were evaluated using a 5 stage scale (1: none; 2: mild; 3: moderate; 4: severe; and 5: extremely severe) ${ }^{28,31}$ We also evaluated the changes in their weekly mean values. Furthermore, we evaluated QOL and CCS scores before and after drug administration using a questionnaire. QOL was evaluated in terms of defecation-related QOL and health-related QOL. The JPAC-QOL was used to evaluate defecation-related QOL. This questionnaire consists of a total of 28 items, including 4 domains related to constipation (physical discomfort, psychological discomfort, worries/concerns, and satisfaction) and their subscales, and is used to evaluate symptoms from the previous 2 weeks. ${ }^{21-23}$ For health-related QOL, we used the SF-8 questionnaire to evaluate the 8 items of physical functioning, role physical, bodily pain, general health, vitality, social functioning, role emotional and mental health, as well as the physical component summary (PCS) score and the mental component summary (MCS) score. ${ }^{19,20}$ The CSS questionnaire evaluated the number of bowel movements, difficulty in defecating, sensation of incomplete evacuation, abdominal pain, defecation time, presence or absence of assistance with defecation, number of failed attempts to defecate despite toileting, and duration of defecation disorder based on a 5 stage scale. ${ }^{20}$ The results of these evaluations were used to compare the effect of $\mathrm{MgO}$ versus placebo 
in the 2 groups.

\section{Sample Size Determination}

To date, 2 studies have detailed overall symptom improvement of constipation with $\mathrm{MgO}$ and reported a $90 \%$ symptom improvement rate. ${ }^{13}$ The overall symptom improvement effects of other agents relative to placebo were recently reported as $17.5 \%$ (linaclotide) $^{32}$ and $29.4 \%$ (lubiprostone). ${ }^{33}$ Since the proportions of placebo and $\mathrm{MgO}$ responders for overall improvement were expected to be $20.0 \%$ and $70.0 \%$, respectively, and the additive effect was expected to be $50.0 \%$, a sample size of 30 (15 patients in each arm) was calculated to provide a one-sided level of significance of $5.0 \%$ and a detection power of $80.0 \%$. Allowing for the dropout of 4 subjects ( 2 subjects per group), a grand total of 34 subjects were included in this study.

\section{Statistical Methods}

All results are expressed as mean \pm standard deviation. Paired $t$ test, Mann-Whitney $U$ test and Fisher's exact test and analysis of covariance were used for comparison of the 2 groups. Statistical significance was defined as a value of $P<0.05$. Statistical analysis was performed using JMP version 13 (JMP Pro; SAS Institute Inc, State of North Carolina, IL, USA).

\section{Results}

\section{Enrollment and Baseline Characteristics of the Patients}

The 34 enrolled patients with $\mathrm{CC}$ were randomly allocated to the $\mathrm{MgO}$ group or placebo group ( $\mathrm{n}=17$ each). Of the randomized subjects, we analyzed the data from 33 patients, since 1 patient who failed to complete the medication regimen was excluded from the final analysis. Thus, data from 16 placebo patients and $17 \mathrm{MgO}$ patients were analyzed. The characteristics of the enrolled patients are shown in Table 1. The patient flowchart is summarized in Figure 2 .

\section{Effect of Magnesium Oxide on Overall Symptom Improvement in Japanese Patients With Chronic Constipation}

Compared to the placebo group, the $\mathrm{MgO}$ group had significantly lower overall improvement score in each week (week $1, P<$ 0.001 ; week 2, $P<0.001$; week $3, P=0.002$; and week $4, P<$ 0.001 ) (Fig. 3A). Furthermore, compared to the response rate of $25.0 \%$ for overall symptom improvement in the placebo group, that

Table 1. Characteristics of the Patients

\begin{tabular}{lccc}
\hline \multicolumn{1}{c}{ Variable } & Placebo & Magnesium oxide & $P$-value \\
\hline Sex (male/female) & $0 / 16$ & $0 / 17$ & 0.271 \\
Age (yr) & $42.9 \pm 12.7$ & $39.0 \pm 12.9$ & 0.357 \\
Height (cm) & $159.6 \pm 5.1$ & $157.4 \pm 6.4$ & 0.471 \\
Weight (kg) & $56.4 \pm 10.1$ & $54.0 \pm 8.6$ & 0.986 \\
BMI & $22.1 \pm 3.3$ & $21.7 \pm 2.6$ & 0.601 \\
Alcohol & $2(12.5)$ & $1(5.9)$ & $>0.999$ \\
Smoking & $9(56.3)$ & $1.8 \pm 1.1$ & 0.067 \\
SBM (n/wk) & $2.5 \pm 1.0$ & $0.8 \pm 1.3$ & 0.323 \\
CSBM (n/wk) & $1.3 \pm 1.6$ & $1.8 \pm 0.6$ & 0.221 \\
BSFS score & $2.3 \pm 1.1$ & $75.5 \pm 37.3$ & 0.036 \\
Colon transit time (hr) & $46.4 \pm 36.9$ & $2.2 \pm 1.1$ & 0.673 \\
Abdominal bloating (score) & $2.1 \pm 1.1$ & $1.9 \pm 0.9$ & \\
Abdominal discomfort (score) & $1.9 \pm 1.2$ & $2.0 \pm 1.0$ & \\
Straining (score) & $2.0 \pm 1.1$ & $2.1 \pm 1.1$ & \\
Sensation of incomplete evacuation (score) & $1.6 \pm 0.8$ & $50.6 \pm 4.6$ & 0.993 \\
SF-8 physical component summary (score) & $52.1 \pm 4.9$ & $47.8 \pm 5.5$ &
\end{tabular}

BMI, body mass index; SBM, spontaneous bowel movement; CSBM, complete spontaneous bowel movement; SF-8, short form-8; JPAC-QOL, the Japanese version of the patient assessment of constipation quality of life.

Data are presented as $\mathrm{n}$, mean $\pm \mathrm{SD}$, or $\mathrm{n}(\%)$. 
with $\mathrm{MgO}$ was significantly higher at $70.6 \%(P=0.015)$ (Fig. 3B).

\section{Weekly Average Value of Spontaneous Bowel Movement and Its Change From Baseline}

The weekly average SBM in the $\mathrm{MgO}$ group improved each week compared to baseline; further, the degree of change in weekly average from baseline in the $\mathrm{MgO}$ group was significantly different from that of the placebo group, except during week 3 (week 1, $P=0.029$; week 2, $P=0.027$; week $3, P=0.084$; and week 4, $P=0.007$ ). Additionally, with respect to the degree of change in the mean value during the treatment period relative to the baseline value, the $\mathrm{MgO}$ group showed significantly greater improvement than the placebo group (placebo, $2.86 \pm 2.42 ; \mathrm{MgO}, 6.07 \pm 2.26$; $P=0.002)$ (Fig. 4A).

\section{Weekly Average Value of Complete Spontaneous Bowel Movement and the Response Rate of Complete Spontaneous Bowel Movement}

The average value of CSBM in the $\mathrm{MgO}$ group improved each week as compared to baseline values. However, the degree of change in the weekly average value from the baseline figure was not significantly different from that in the placebo group except during week 4 (week $1, P=0.684$; week $2, P=0.052$; week $3, P=$

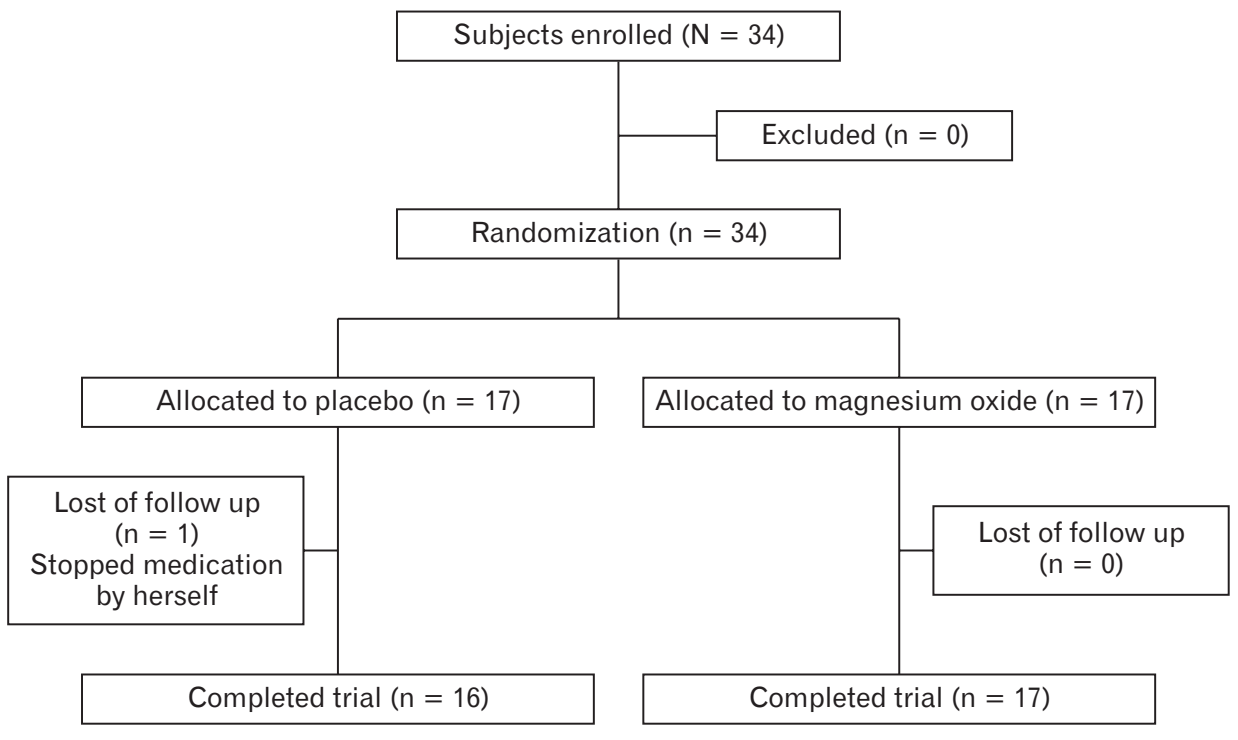

Figure 2. Patient's flowchart summary.

A

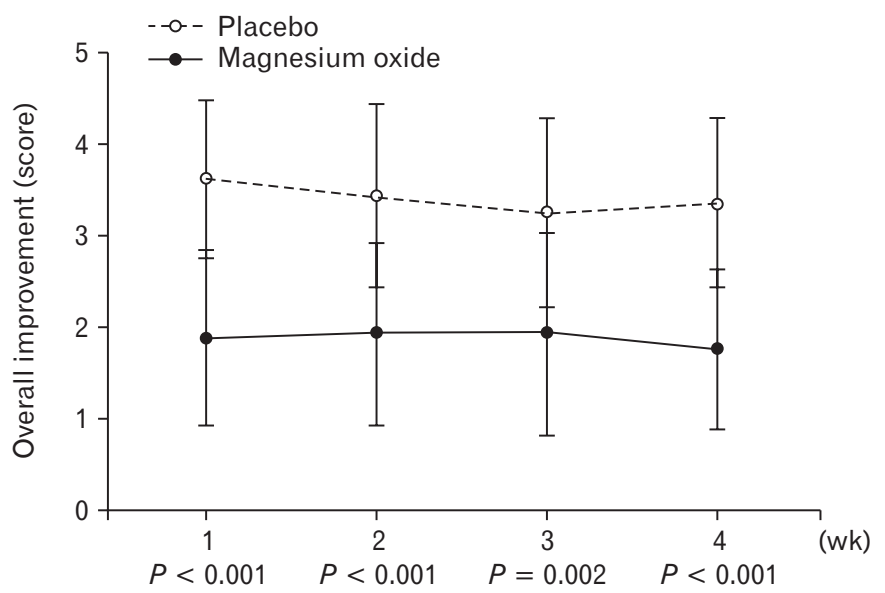

B

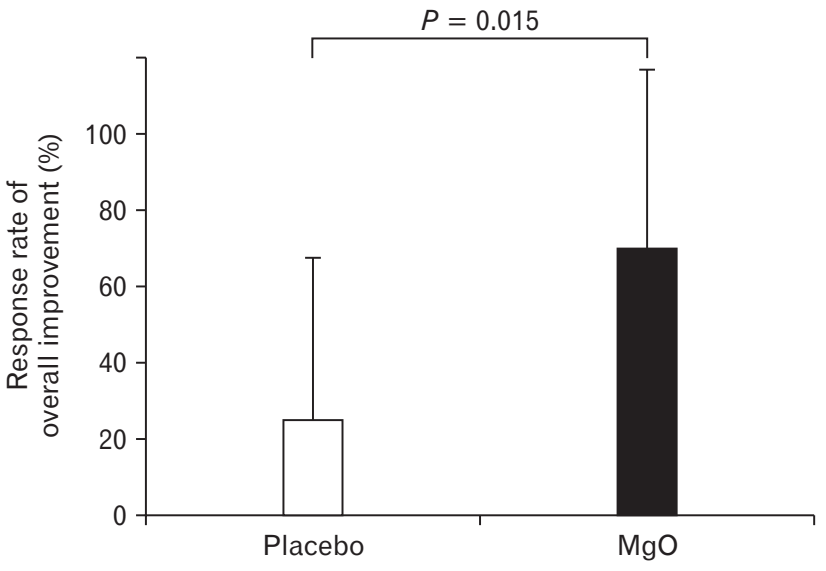

Figure 3. Effect of overall symptomatic improvement in Japanese patients with chronic constipation. (A) Weekly average value of overall improvement score. (B) Response rate of overall improvement. Compared to the response rate of $25.0 \%$ for overall symptom improvement in the placebo group, that with magnesium oxide $(\mathrm{MgO})$ was significantly higher at $70.6 \%$. 
A

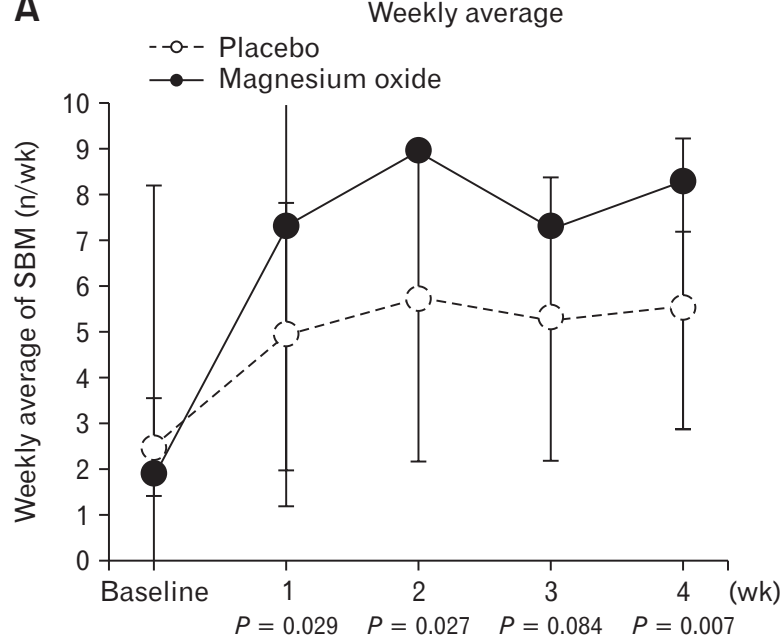

B

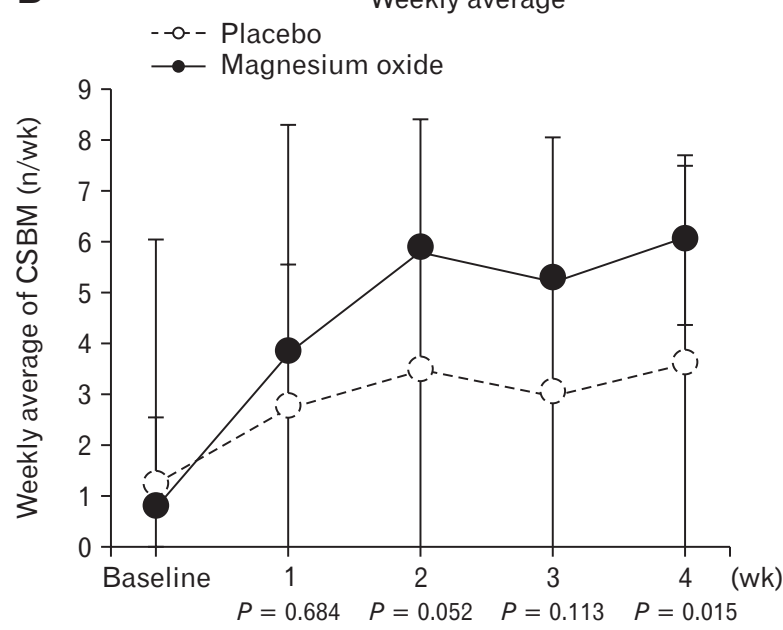

C

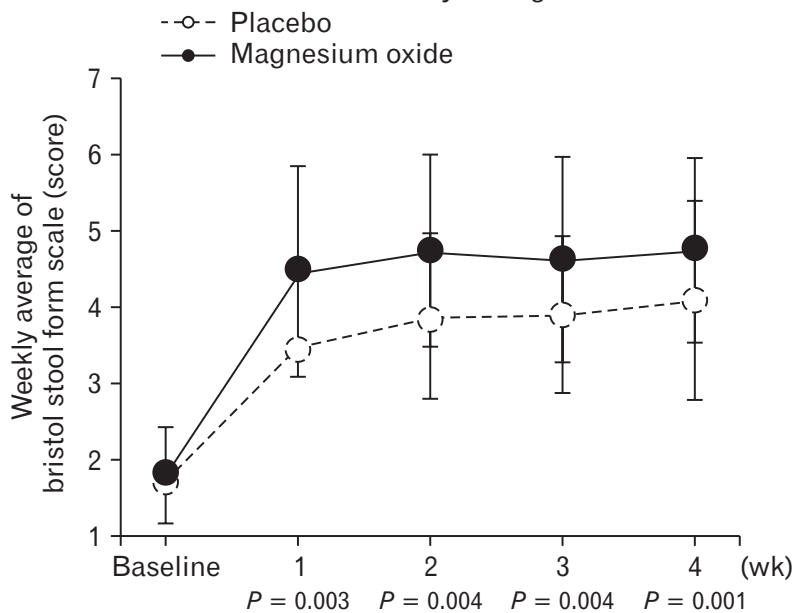

Change from baseline

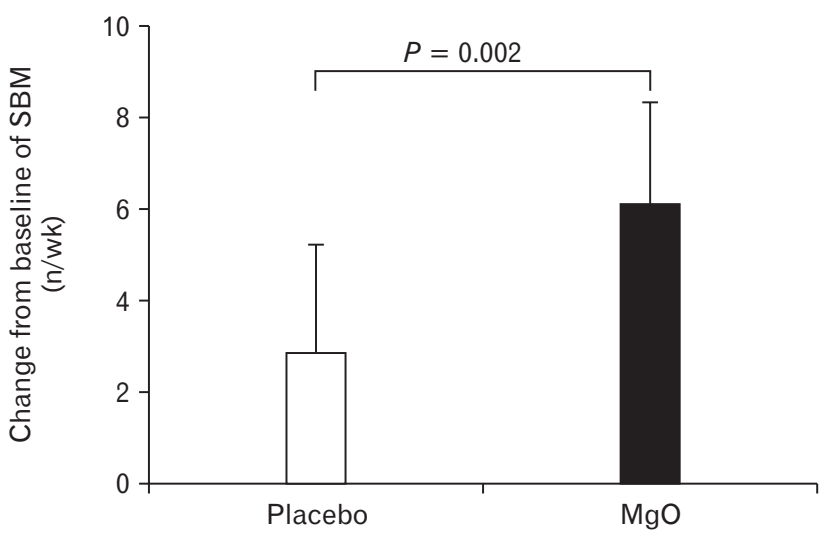

Response rates

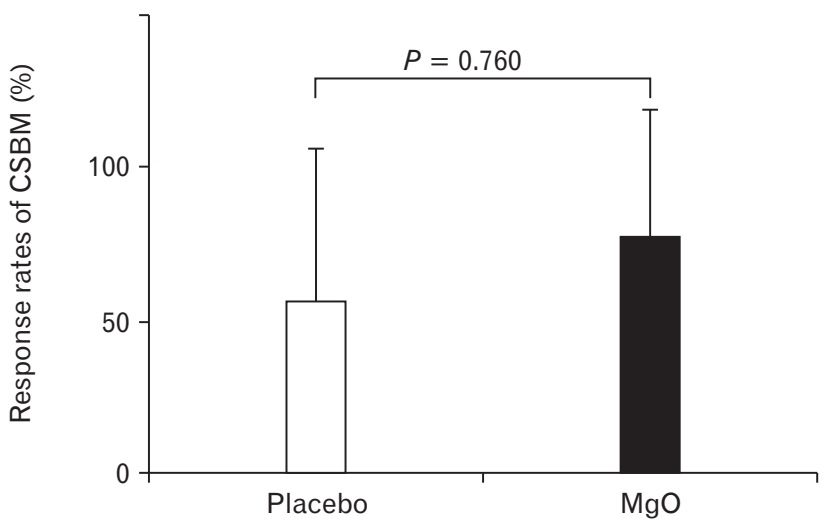

Change from baseline

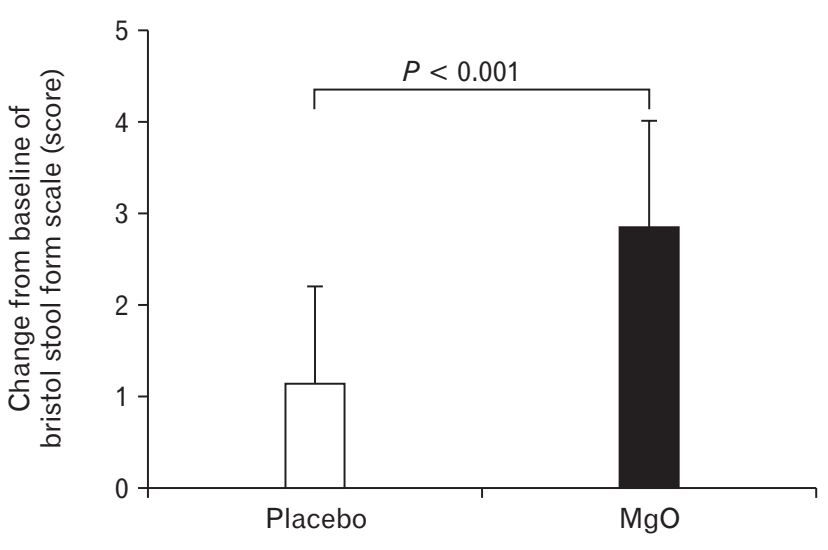

Figure 4. Changes of spontaneous bowel movement (SBM) and Bristol stool form scale (BSFS) after magnesium oxide (MgO) treatment in Japanese patients in chronic constipation. (A) Weekly average value of SBM and its change from baseline. (B) Weekly average value of complete spontaneous bowel movement (CSBM) and the response rate of CSBM. (C) Weekly average of BSFS score and its change from baseline. 
Table 2. Change in Colon Transit Time by Magnesium Oxide in Japanese Patients With Chronic Constipation

\begin{tabular}{|c|c|c|c|c|c|c|}
\hline & \multicolumn{3}{|c|}{ Placebo } & \multicolumn{3}{|c|}{ Magnesium oxide } \\
\hline & Pre & Post & $P$-value & Pre & Post & $P$-value \\
\hline Right colon & $12.3 \pm 9.3$ & $7.0 \pm 6.8$ & 0.063 & $18.5 \pm 10.8$ & $11.4 \pm 7.8$ & 0.029 \\
\hline Left colon & $18.5 \pm 21.1$ & $9.8 \pm 10.6$ & 0.037 & $28.0 \pm 17.3$ & $14.3 \pm 16.3$ & 0.003 \\
\hline Rectosigmoid colon & $16.0 \pm 16.0$ & $14.9 \pm 14.3$ & 0.799 & $28.5 \pm 21.3$ & $20.6 \pm 22.5$ & 0.210 \\
\hline Total colon & $46.4 \pm 36.9$ & $31.6 \pm 25.5$ & 0.109 & $75.5 \pm 37.3$ & $41.6 \pm 30.5$ & $<0.001$ \\
\hline
\end{tabular}

Data are presented as mean $\pm \mathrm{SD}$.

A

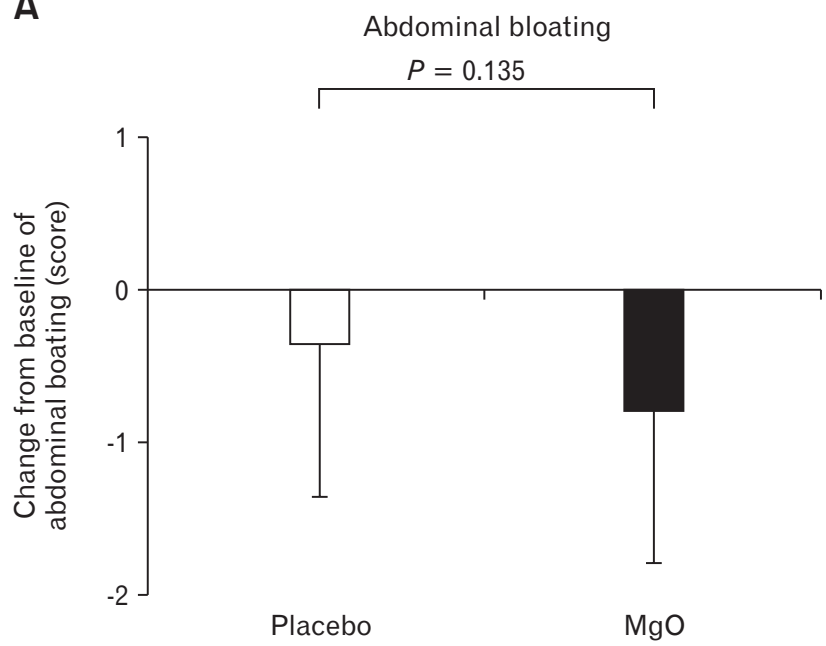

C

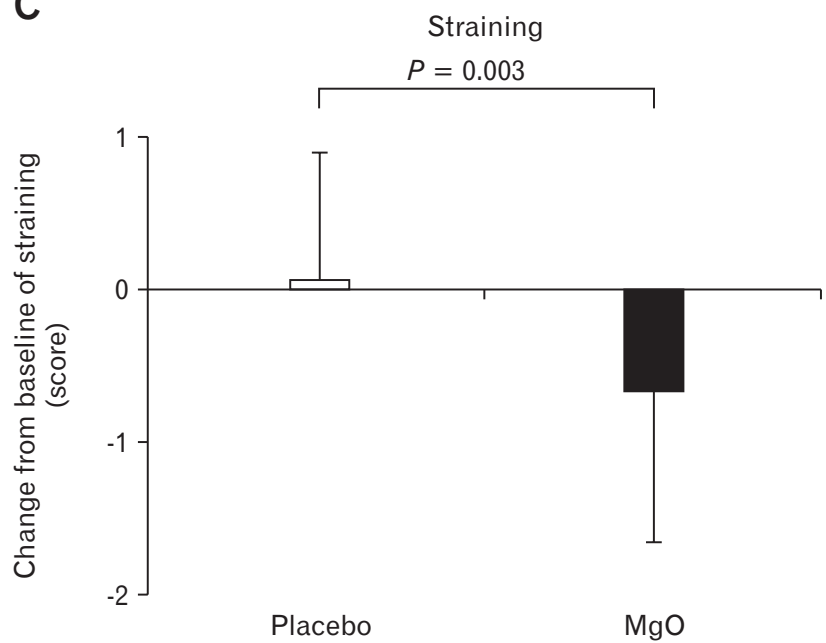

B

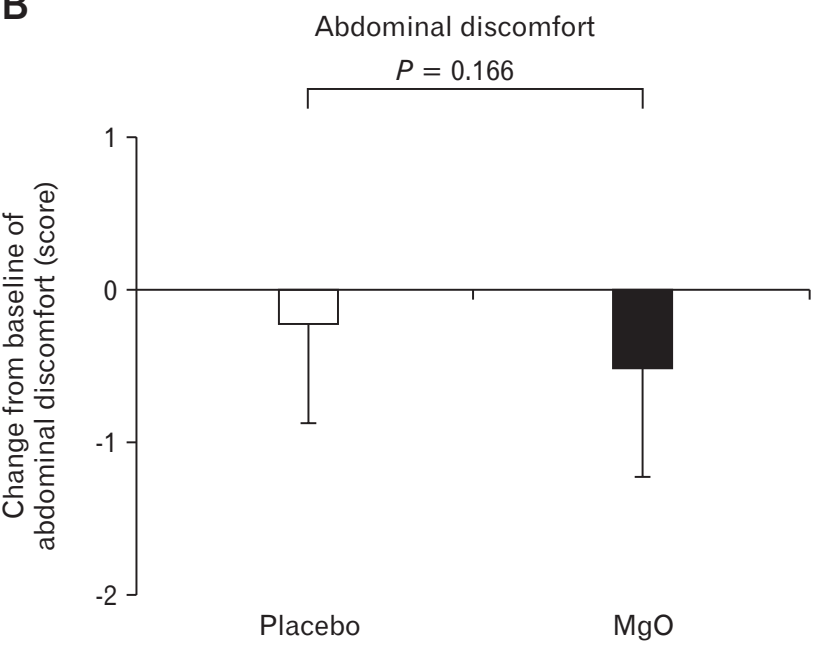

D

Sensation of incomplete evacuation $P=0.329$

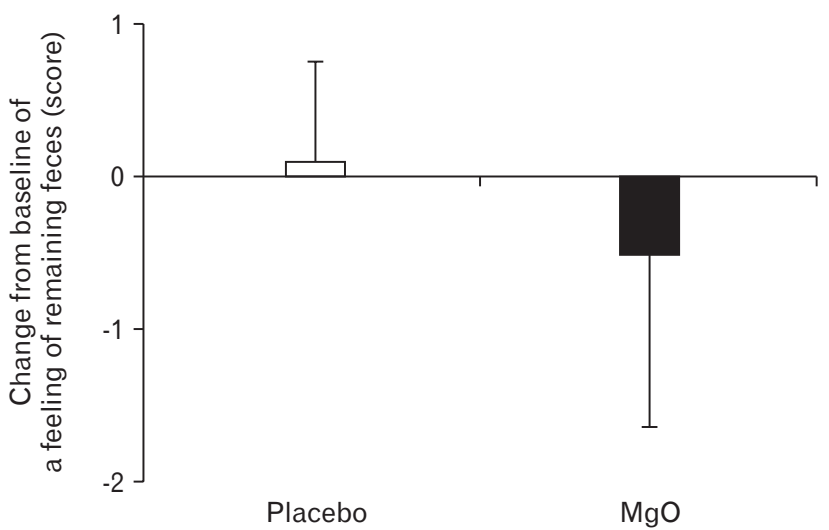

Figure 5. Effect of magnesium oxide (MgO) on gastrointestinal symptoms in Japanese patients with chronic constipation. (C) The severity score for straining during defecation in the placebo group did not differ before versus after taking the drug, although there was significant improvement in the $\mathrm{MgO}$ group $(P=0.003)$. The 2 groups did not show any statistically significant differences in terms of $(\mathrm{A})$ abdominal bloating, $(\mathrm{B})$ abdominal discomfort, or (D) sensation of incomplete evacuation. 
0.113; week 4, $P=0.015)$. We observed no significant difference between the $\mathrm{MgO}$ and placebo groups in terms of overall CSBM response rates (placebo, $56.3 \pm 49.6 \%$; $\mathrm{MgO}, 76.5 \pm 42.4 \% ; P=$ 0.760) (Fig. 4B).

\section{Weekly Average Value of Bristol Stool Form Scale Score and Its Change From Baseline}

The weekly average of BSFS score in the $\mathrm{MgO}$ group improved each week as compared to the baseline value, and the degree of change in the average scores compared to baseline values were significantly different from those in the placebo group (week 1, $P$ $=0.003$; week $2, P=0.004$; week $3, P=0.004$; week $4, P=$
0.001). Furthermore, the degree of change in average scores from the baseline value during the treatment period suggested that stools were softened significantly more often in the $\mathrm{MgO}$ group than in the placebo group (placebo, $1.15 \pm 1.03 ; \mathrm{MgO}, 2.83 \pm 1.15 ; P<$ 0.001) (Fig. 4C).

\section{Change in Colon Transit Time by Magnesium Oxide in Japanese Patients With Chronic Constipation}

While placebo administration did not improve total CTT $(P=$ $0.109), \mathrm{MgO}$ administration significantly improved total CTT ( $P$ $<0.001)$.

Regarding the CTT of segmental colon, $\mathrm{MgO}$ administration

\section{A SF-8}
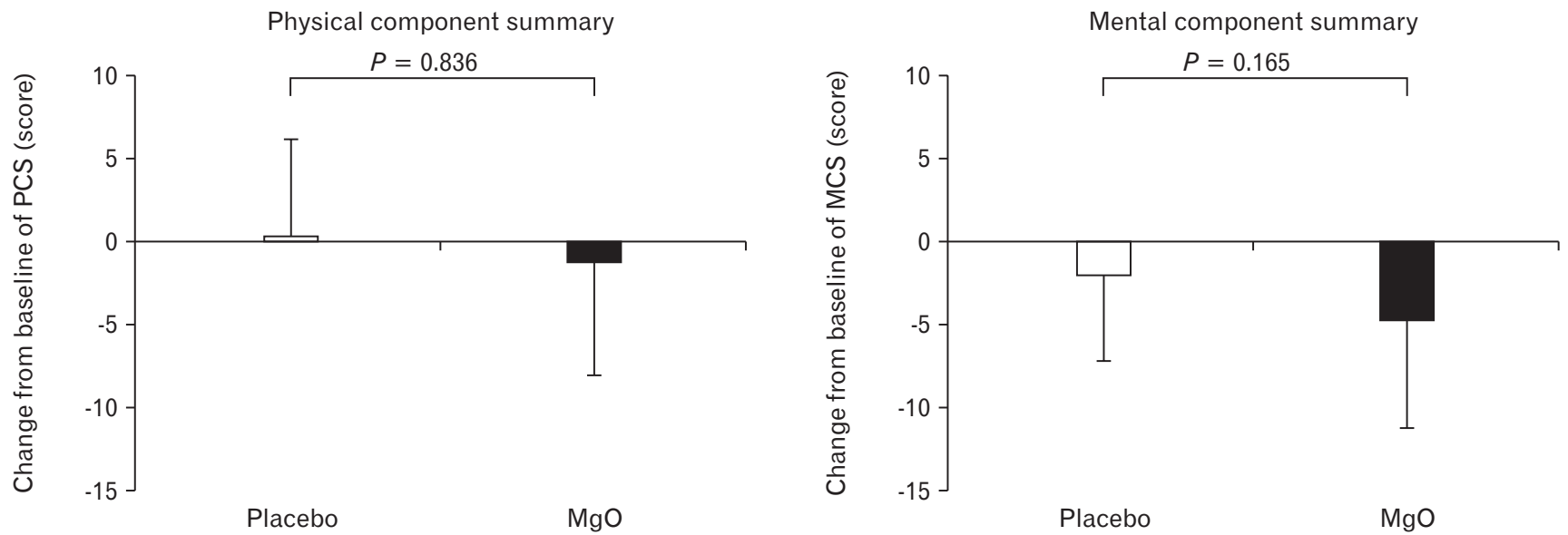

B JPAC-QOL

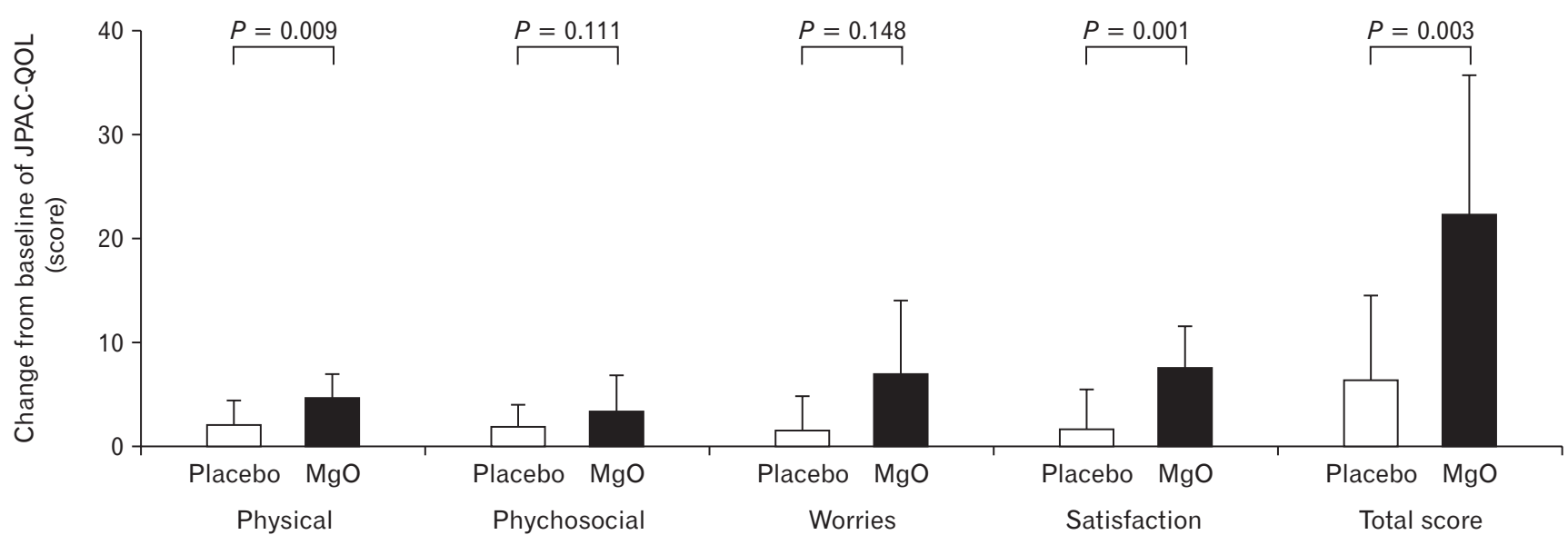

Figure 6. Change of quality of life ([A] short form-8 [SF-8] and the [B] Japanese version of the patient assessment of constipation quality of life [JPACQOL]) in Japanese patients with chronic constipation. The 2 groups also showed no differences in physical component summary (PCS) score and mental component summary (MCS) score. JPAC-QOL evaluations indicated that the degree of change in the total score before versus after the intervention was significantly higher in the magnesium oxide $(\mathrm{MgO})$ group than in the placebo group. 
significantly improved in the right colon $(P=0.029)$ and the left colon $(P=0.003)$, however there was no statistical difference in the rectosigmoid colon $(P=0.210)$ (Table 2$)$.

\section{Effect of Magnesium Oxide on Gastrointestinal Symptoms (Abdominal Bloating, Abdominal Discomfort, Straining, and Sensation of Incomplete Evacuation)}

The severity score for straining during defecation in the placebo group did not differ before versus after taking the drug, although there was significant improvement in the $\mathrm{MgO}$ group (placebo, 0.06 $\pm 0.83 ; \mathrm{MgO},-0.65 \pm 1.00 ; P=0.003)$. The 2 groups did not show any statistically significant differences in terms of abdominal bloating (placebo, $-0.34 \pm 1.02 ; \mathrm{MgO},-0.79 \pm 0.99 ; P=0.135$ ), abdominal discomfort (placebo, $-0.22 \pm 0.65 ; \mathrm{MgO},-0.50 \pm$ $0.72 ; P=0.166$ ), or sensation of incomplete evacuation (placebo, $0.09 \pm 0.67 ; \mathrm{MgO},-0.51 \pm 1.14 ; P=0.329$ ) (Fig. 5).

\section{Change From Baseline of Quality of Life (Short Form-8 and the Japanese Version of the Patient Assessment of Constipation Quality of Life) in Japanese Patients With Chronic Constipation}

Evaluations using SF-8 demonstrated that the 2 groups did not show any statistically significant differences in terms of physical functioning (placebo, $-0.54 \pm 5.09 ; \mathrm{MgO},-0.59 \pm 5.93 ; P=$ 0.722 ), role physical (placebo, $-0.67 \pm 5.93 ; \mathrm{MgO},-0.70 \pm 6.47$; $P=0.890$ ), bodily pain (placebo, $-0.71 \pm 7.33 ; \mathrm{MgO},-4.23 \pm$ $6.40 ; P=0.159$ ), general health (placebo, $-3.72 \pm 5.35 ; \mathrm{MgO}$, $-6.26 \pm 6.09 ; P=0.273$ ), vitality (placebo, $-0.29 \pm 5.56 ; \mathrm{MgO}$, $-3.55 \pm 6.66 ; P=0.435$ ), social functioning (placebo, $-1.67 \pm$ $7.92 ; \mathrm{MgO},-1.12 \pm 8.02 ; P=0.409$ ), role emotional (placebo, $-0.69 \pm 3.98 ; \mathrm{MgO},-3.11 \pm 5.86 ; P=0.155)$, and mental health (placebo, $-3.79 \pm 8.04 ; \mathrm{MgO},-6.12 \pm 5.6 ; P=0.188$ ) before versus after taking the drug. The 2 groups also showed no differences in PCF score (placebo, $0.20 \pm 5.92 ; \mathrm{MgO},-1.29 \pm$ 6.82; $P=0.836$ ) and MCS score (placebo, $-1.99 \pm 5.13 ; \mathrm{MgO}$, $-4.72 \pm 6.46 ; P=0.165)$.

JPAC-QOL evaluations indicated that the degree of change in the total score before versus after the intervention was significantly higher in the $\mathrm{MgO}$ group than in the placebo group (placebo, $6.31 \pm 8.15 ; \mathrm{MgO}, 22.29 \pm 13.13 ; P=0.003)$. Changes in the domains of physical discomfort (placebo, $1.88 \pm 2.42 ; \mathrm{MgO}, 4.47$ $\pm 2.38 ; P=0.009$ ) and satisfaction (placebo, $1.31 \pm 4.07 ; \mathrm{MgO}$, $7.47 \pm 3.91 ; P=0.001)$ were significantly different between the 2 groups, whereas psychological discomfort (placebo, $1.69 \pm 2.20$;

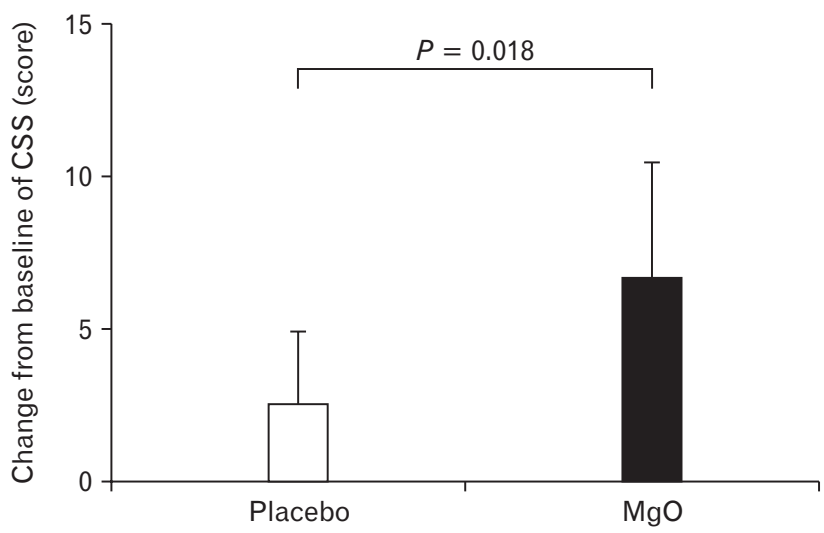

Figure 7. Change in the constipation scoring system (CSS) score in Japanese patients with chronic constipation. While administration of magnesium oxide $(\mathrm{MgO})$ significantly improved defecation status, we did not observe any improvement in the placebo group $(P=0.018)$.

$\mathrm{MgO}, 3.35 \pm 3.34 ; P=0.111$ ) and worries/concerns (placebo, $1.44 \pm 3.16 ; \mathrm{MgO}, 7.00 \pm 7.07 ; P=0.148)$ were not different (Fig. 6).

\section{Change in the Constipation Scoring System Score in Japanese Patients With Chronic Constipation}

While administration of $\mathrm{MgO}$ significantly improved defecation status, we did not observe any improvement in the placebo group (placebo, 2.56 $\pm 2.42 ; \mathrm{MgO}, 6.65 \pm 3.71 ; P=0.018$ ) (Fig. 7).

\section{Discussion}

Although $\mathrm{MgO}$ is a medicine for $\mathrm{CC}$ that is used by approximately 10 million people in Japan, ${ }^{11}$ to date no studies have objectively examined its effects. The current single-site doubleblind controlled trial examined the effect of administration of $\mathrm{MgO}$ capsules (1.5 $\mathrm{g}$ daily) versus placebo capsules on overall symptoms of constipation, CTT, SBM, stool form, and QOL of Japanese patients with mild to moderate $\mathrm{CC}$. Administration of $\mathrm{MgO}$ for 4 weeks improved overall constipation symptoms, shortened CTT, softened stool form, and improved constipation-specific QOL scores.

$\mathrm{CC}$ is significantly influenced by psychological factors; evaluation of the clinical effect of a drug while eliminating psychological factors requires comparisons with a placebo. In recent years, the concept of PRO has been spreading mainly in Western countries and it has become an essential part of clinical studies and future diagnostics; the importance of PRO has been recognized even in the 
diagnosis and treatment of gastroenterological diseases. ${ }^{14,15}$ PRO is an approach in which the patient himself/herself directly evaluates the usefulness of a treatment. In the present study, it was meaningful to evaluate the efficacy of the drug by obtaining complete questionnaires from the patients with constipation.

$\mathrm{MgO}$ is an osmotic laxative that is low in cost and has few adverse effects. In Japan, it is used as a first-line drug in actual clinical practice. It is minimally absorbed in the gastrointestinal tract, raises the osmotic pressure in the intestinal tract, and increases the frequency of defecation by causing water secretion in the lumen of the intestinal tract. Presently, it is recommended as a first priority according to the 2017 Japanese guidelines for diagnosis and treatment of chronic constipation as 1 , with an evidence level of A. ${ }^{12}$ However, since there has not been a large-scale clinical trial on $\mathrm{MgO}$ within or outside of Japan to date, the recommendation grade according to the ACG diagnosis and treatment guidelines remains $\mathrm{B} .{ }^{34}$ For this reason, this study has clinical significance since it is the first study to compare the therapeutic effect of $\mathrm{MgO}$ with a placebo drug and to examine it objectively.

In this study, we first examined the number of SBM and CSBM before and after administering $\mathrm{MgO}$. With respect to SBM, it was reported in clinical studies involving Japanese subjects that the administration of lubiprostone and linaclotide significantly increases the number of bowel movements compared to placebo administration. ${ }^{28,35,36}$ In the present study, where $1.5 \mathrm{~g} /$ day of $\mathrm{MgO}$ was administered for 4 weeks, the mean number of bowel movements per week after placebo administration was about 3 , while that in the $\mathrm{MgO}$ group increased to around 6. Compared to the results of clinical studies using other agents, this result showed this drug can cause a clear increase in the number of bowel movements in patients with mild to moderate $\mathrm{CC}$.

Furthermore, the fact that placebo administration also resulted in a clear increase in the number of bowel movements from immediately after administration, thereby once again demonstrating the placebo effect in constipation patients, is an interesting result. We also examined the response rate to CSBM, which has been gaining attention in recent times as an indicator of constipation. In Japan, reports from phase III clinical studies of linaclotide and elobixibat indicated that the response rate of CSBM relative to placebo after drug administration for 1 week was $31.7 \%$ vs $10.0 \%$ and $52.2 \%$ vs $17.5 \%$, respectively, meaning the response rates were significantly higher in the actual drug groups. ${ }^{28,37}$ On the other hand, in this study, the response rates of $\mathrm{MgO}$ and placebo administrations were $76.0 \%$ and $56.0 \%$, respectively, representing no statistically significant differences between the 2 results.
However, the fact that the response rate of the placebo was clearly higher than the results of clinical trials on linaclotide and elobixibat may have been significantly affected by the fact that this study involved patients with mild to moderate constipation.

The present study also examined the influence of $\mathrm{MgO}$ administration on abdominal symptoms. We found that $\mathrm{MgO}$ administration softened the stools and significantly reduced straining while defecating. However, there were no improvements in abdominal bloating, abdominal discomfort, and sensation of incomplete evacuation. On the other hand, in the phase III clinical trial of lubiprostone in Japan, all 4 symptoms, namely straining while defecating, abdominal bloating, abdominal discomfort, and sensation of incomplete evacuation, improved: even linaclotide reportedly results in improvements in the passage of stools as well as abdominal pain and discomfort. $^{28,36,38,39}$

$\mathrm{MgO}$ is an osmotic laxative, whereas lubiprostone is a drug that facilitates water secretion into the intestine and induces bowel movement by activating the chloride channels that exist in the small intestinal epithelium. ${ }^{33,35,36,38}$ In contrast, linaclotide induces defecation by acting on the guanylate cyclase $\mathrm{C}$ receptors that exist on the surface of intestinal epithelial cells and raising the concentration of cGMP in the intestinal tract to facilitate the secretion of water into the intestine. ${ }^{27,28,32,39} \mathrm{cGMP}$ also inhibits the afferent nerves of the intestinal submucosal tissue and is thus reported to have the effect of lessening abdominal pain.

In recent years, evaluation of CSBM has been considered important in clinical studies, but to achieve SBM with no sensation of incomplete evacuation, it is important to encourage type 4 stool form according to the BSFS. According to the results of the current study, use of $1.5 \mathrm{~g} /$ day of $\mathrm{MgO}$ produced average BSFS score of 4.6, and stool softening was slightly greater with $\mathrm{MgO}$ than lubiprostone (3.4 to 3.8$).{ }^{35}$ Given these facts, it may explain why treatment with $\mathrm{MgO}$ did not result in symptom improvement.

The 2017 Japanese guidelines for diagnosis and treatment of constipation describe that $\mathrm{CC}$ is classified as slow transit constipation, normal transit constipation, or functional defecation disorder according to its pathology. ${ }^{12}$ However, there are almost no data on measurement of CTT with the existing laxatives, with the only relevant report being that of lubiprostone. Lubiprostone, a chloride channel activator introduced in 2012, has been reported to significantly shorten CTT and improve constipation symptoms. According to the study by Christie et al, ${ }^{33}$ administration of $48 \mu \mathrm{g} /$ day of lubiprostone improved colonic transit time by 19.9 hours compared to placebo.

In the present examination as well, we measured CTT using 
an opaque marker, which demonstrated that administration of 1.5 $\mathrm{g} /$ day of $\mathrm{MgO}$ shortened the CTT by 19.1 hours. Based on this result, it is conjectured that $\mathrm{MgO}$ softens stool by drawing water from the intestinal wall, stimulates the intestine by increasing the stool mass, and shortens CTT. This interesting result provides supporting data for the observed increase in the number of bowel movements.

In this study, we also studied the QOL of patients with constipation. Reportedly, patients with $\mathrm{CC}$ experience decreased QOL. ${ }^{40}$ It has also been reported that administration of lubiprostone improves QOL in terms of PCS and MCS, while linaclotide improves the QOL of constipated patients with irritable bowel syndrome. ${ }^{28}$ Here, we evaluated QOL using 2 indicators, namely the general SF-8 QOL scale and the constipation-specific QOL scale of JAC-QOL. While JPAC-QOL showed a significant improvement in QOL through $\mathrm{MgO}$ administration, $\mathrm{SF}-8$ scale scores revealed no statistically significant difference in QOL before versus after drug administration. The design of this study was such that $\mathrm{MgO}$ was taken for 4 weeks, which was a shorter period than that in reports that have traditionally reported improvements in QOL. In general, improvements in QOL are known to occur more slowly than improvements in symptoms, and our observation of a difference in the symptom-based questionnaire of JPAC-QOL, and lack of an improvement in the general QOL indicator of SF-8, may have been affected by the relatively short observation period. Moreover, the fact that this study enrolled patients with mild to moderate constipation meant that the baseline QOL may not have been much lower than that of healthy adults, which in turn could have affected the results of the study.

A limitation of this study was its single-site setting including patients with constipation who were recruited by the newspaper advertisement. All were female patients with a mean age of 40 years, and a relatively small number of subjects (34 subjects in total) were enrolled. Furthermore, female patients generally experience a greater placebo effect than male patients, and because this study examined patients with mild to moderate constipation, we cannot rule out the possibility of patient bias. Since this study exhibits a clearly higher placebo effect than the results of recent phase III clinical studies of other agents involving Japanese patients, it may explain why the CSBM results were negative. A higher proportion of females than males complain of constipation symptoms, with the prevalence in males reportedly increasing with age. Recent internet investigations also showed a significant trend in constipation among young women, and it has been reported that women tend to have fewer number of bowel movements than men. ${ }^{41}$ As our present study examined only female patients approximately 40 years of age, further studies are needed to understand the effect of $\mathrm{MgO}$ that will be seen in elderly patients. Moreover, we did not perform the anorectal manometry to exclude patients with defecatory disorders for enrolled subjects. However, there were no cases that remains opacifying markers only in the rectosigmoid colon. In addition, we did not enroll subjects who have severe sensation of incomplete evacuation.

Here, we performed the first randomized double-blind placebo-controlled parallel-group study to evaluate the therapeutic effect of $\mathrm{MgO}$ in Japanese patients with mild to moderate CC. The results indicate that administration of $\mathrm{MgO}$ significantly improved the defecation status of $\mathrm{CC}$ patients. $\mathrm{MgO}$ also significantly shortened CTT and improved the number of bowel movements, stool form, and constipation-specific QOL. We believe that further studies with greater numbers of subjects are needed to validate our findings.

\section{Financial support: None.}

\section{Conflicts of interest: None.}

Author contributions: Sumire Mori and Toshihiko Tomita contributed to the concept and design of the study, were involved in data acquisition and analysis, and provided statistifical design; Sumire Mori, Toshihiko Tomita, Kazuki Fujimura, Haruki Asano, Tomohiro Ogawa, Takahisa Yamasaki, Takashi Kondo, Tomoaki Kono, Katsuyuki Tozawa, Tadayuki Oshima, Hirokazu Fukui, Takeshi Kimura, Jiro Watari, and Hiroto Miwa contributed to data interpretation, critically reviewed the manuscript for important intellectual contents, and approved final version of the manuscript; and Hiroto Miwa coordinated acquisition of data and trial supervision.

\section{References}

1. Bharucha AE, Pemberton JH, Locke GR 3rd. American gastroenterological association technical review on constipation. Gastroenterology 2013;144:218-238.

2. Tack J, Müller-Lissner S, Stanghellini V, et al. Diagnosis and treatment of chronic constipation a European perspective. Neurogastroenterol Motil 2011;23:697-710

3. Gray JR. What is chronic constipation? Definition and diagnosis. Can J Gastroenterol 2011;25(suppl B):7B-10B.

4. Chatoor D, Emmnauel A. Constipation and evacuation disorders. Best Pract Res Clin Gastroenterol 2009;23:517-530.

5. Lembo A, Camilleri M. Chronic constipation. N Engl J Med 2003;349:1360-1368 
6. Pemberton JH, Rath DM, Ilstrup DM. Evaluation and surgical treatment of severe chronic constipation. Ann Surg 1991;214:403-411; discussion 411-413.

7. Mertz H, Naliboff B, Mayer E. Physiology of refractory chronic constipation. Am J Gastroenterol 1999;94:609-615.

8. Lacy BE, Levenick JM, Crowell M. Chronic constipation: new diagnostic and treatment approaches. Therap Adv Gastroenterol 2012;5:233247.

9. Tillou J, Poylin V. Functional disorders: slow-transit constipation. Clin Colon Rectal Surg 2017;30:76-86.

10. Alame AM, Bahna H. Evaluation of constipation. Clin Colon Rectal Surg 2012;25:5-11.

11. Ministry of Health, Labour and Walfare. Hypermagnesaemia caused by magnesium oxide. Available from URL: https://www.pmda.go.jp/ english/safety/info-services/drugs/medical-safety-information/0013.html (accessed 3 Sep 2019). Pharmaceuticals and Medical Devices Safety Information 2015;328:5-8.

12. The Japanese Society of Gastroenterology. Evidence-based Clinical Practice Guidelines for Chronic Constipation 2017, ed. Research society for the Diagnosis and Treatment of Chronic Constipation, Affiliated to The Japanese Society of Gastroenterology in Japanese, Tokyo:Nankodo Co, Ltd. 2017.

13. Sakimura K, Machino H, Miyoshi K, et al. Clinical evaluation of YO106 (magnesium oxide tablets) in the treatment of habitual constipationa cross over study with magnesium oxide powders. Jpn Pharmacol Ther 1998;26:1027-1053.

14. Basch E, Rogak LJ, Dueck AC. Methods for implementing and reporting patient-reported outcome (PRO) measures of symptomatic adverse events in cancer clinical trials. Clin Ther 2016;38:821-830.

15. Donovan JL, Hamdy FC, Lane JA, et al. Patient-reported outcomes after monitoring, surgery, or radiotherapy for prostate cancer. $\mathrm{N}$ Engl J Med 2016;375:1425-1437.

16. Lacy BE, Mearin F, Chang L, et al. Bowel Disorders. Gastroenterology 2016;150:1393-1407, e5.

17. Horibata K, Tanoue A, Ito M, Takemura Y. Relationship between renal function and serum magnesium concentration in elderly outpatients treated with magnesium oxide. Geriatr Gerontol Int 2016;16:600-605.

18. Yamasaki M, Funakoshi S, Matsuda S, et al. Interaction of magnesium oxide with gastric acid secretion inhibitors in clinical pharmacotherapy. Eur J Clin Pharmacol 2014;70:921-924.

19. Turner-Bowker DM, Bayliss MS, Ware JE Jr, Kosinski M. Usefulness of the SF-8 health survey for comparing the impact of migraine and other conditions. Qual Life Res 2003;12:1003-1012.

20. Tokuda Y, Okubo T, Ohde S, et al. Assessing items on the SF-8 Japanese version for health-related quality of life: a psychometric analysis based on the nominal categories model of item response theory. Value Health 2009;12:568-573.

21. Nomura H, Agatsuma T, Mimura T. Validity and reliability of the Japanese version of the patient assessment of constipation quality of life questionnaire. J Gastroenterol 2014;49:667-673.

22. Tsunoda A, Yamada K, Takano M, Kusanagi H. The translation and validation of the Japanese version of the patient assessment of constipation quality of life scale. Surg Today 2016;46:414-421.

23. Marquis P, De La Loge C, Dubois D, McDermott A, Chassany O. Development and validation of the patient assessment of constipation quality of life questionnaire. Scand J Gastroenterol 2005;40:540-551.

24. Agachan F, Chen T, Pfeifer J, Reissman P, Wexner SD. A constipation scoring system to simplify evaluation and management of constipated patients. Dis Colon Rectum 1996;39:681-685.

25. Cummings JH, Wiggins HS. Transit through the gut measured by analysis of a single stool. Gut 1976;17:219-223.

26. Metcalf AM, Phillips SF, Zinsmeister AR, MacCarty RL, Beart RW, Wolff BG. Simplified assessment of segmental colonic transit. Gastroenterology 1987;92:40-47.

27. Rao S, Lembo AJ, Shiff SJ, et al. A 12-week, randomized, controlled trial with a 4-week randomized withdrawal period to evaluate the efficacy and safety of linaclotide in irritable bowel syndrome with constipation. Am J Gastroenterol 2012;107:1714-1724.

28. Fukudo S, Miwa H, Nakajima A. A randomized controlled and longterm linaclotide study of irritable bowel syndrome with constipation patients in Japan. Neurogastroenterol Motil 2018;30:e13444.

29. O'Donnell LJ, Virjee J, Heaton KW. Detection of pseudodiarrhoea by simple clinical assessment of intestinal transit rate. BMJ 1990;300:439440 .

30. Longstreth GF, Thompson WG, Chey WD, Houghton LA, Mearin F, Spiller RC. Functional bowel disorders. Gastroenterology 2006;130:1480-1491.

31. Chey WD, Camilleri M, Chang L, Rikner L, Graffner H. A randomized placebo-controlled phase IIb trial of a3309, a bile acid transporter inhibitor, for chronic idiopathic constipation. Am J Gastroenterol 2011;106:1803-1812.

32. Lembo AJ, Schneier HA, Shiff SJ, et al. Two randomized trials of linaclotide for chronic constipation. N Engl J Med 2011;365:527-536.

33. Christie J, Shroff S, Shahnavaz N, et al. A randomized, double-blind, placebo-controlled trial to examine the effectiveness of lubiprostone on constipation symptoms and colon transit time in diabetic patients. Am J Gastroenterol 2017;112:356-364.

34. American College of Gastroenterology Chronic Constipation Task Force. An evidence-based approach to the management of chronic constipation in North America. Am J Gastroenterol 2005;100(suppl 1):S1-S4.

35. Fukudo S, Hongo M, Kaneko H, Ueno R. Efficacy and safety of oral lubiprostone in constipated patients with or without irritable bowel syndrome: a randomized, placebo-controlled and dose-finding study. Neurogastroenterol Motil 2011;23:544-e205.

36. Fukudo S, Hongo M, Kaneko H, Takano M, Ueno R. Lubiprostone increases spontaneous bowel movement frequency and quality of life in patients with chronic idiopathic constipation. Clin Gastroenterol Hepatol 2015;13:94-301, e5.

37. Nakajima A, Seki M, Taniguchi S, et al. Safety and efficacy of elobixibat for chronic constipation: results from a randomised, double-blind, placebo-controlled, phase 3 trial and an open-label, single-arm, phase 3 trial. Lancet Gastroenterol Hepatol 2018;3:537-547.

38. Johanson JF, Morton D, Geenen J, Ueno R. Multicenter, 4-week, double-blind, randomized, placebo-controlled trial of lubiprostone, a 
locally-acting type- 2 chloride channel activator, in patients with chronic constipation. Am J Gastroenterol 2008;103:170-177.

39. Rao SS, Quigley EM, Shiff SJ, et al. Effect of linaclotide on severe abdominal symptoms in patients with irritable bowel syndrome with constipation. Clin Gastroenterol Hepatol 2014;12:616-623.

40. Wald A, Scarpignato C, Kamm MA, et al. The burden of constipation on quality of life: results of multinational survey. Aliment Pharmacol Ther 2007;26:227-236.

41. Tamura A, Tomita T, Oshima T. Prevalence and self-recognition of chronic constipation: results of an internet survey. J Neurogastroenterol Motil 2016:30;22:677-685. 